Visualizing Objects, Places, and Spaces: A Digital Project Handbook

\title{
Documentation for Dimensional Projects
}

Beth Fischer, Hannah Jacobs, Jayde Rice

Published on: Oct 14, 2019

DOI: $10.21428 / 51$ bee781.608ce9b9

License: Creative Commons Attribution 4.0 International License (CC-BY 4.0). 


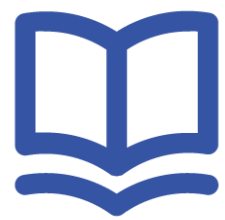

Documentation for dimensional projects serves both internal and external audiences. Even if you are working alone, dimensional projects can be particularly opaque to revisit as you troubleshoot, and it is especially frustrating to make a model work and then not remember what settings you used to get there.

Keeping track of procedures, application settings, mid-project outputs, and the results of ineffective processes will help you troubleshoot efficiently and help you repeat processes that work properly. Once the model is complete, this documentation will also help you write up your methodology for presentations, publications, and it will help you annotate the model for wider audiences. This is critical for models used for both research and presentation purposes, because dimensional projects can introduce a lot of uncertainty about which aspects of the model are created directly from the subject, which are derived from data or outside sources, and which are the researcher's best guess. Dimensional models are compelling because they seem so realistic, but that can also lead other scholars to distrust them or external audiences to trust them too much.

\section{It is a good idea to track:}

\section{- How you acquired content and transferred it to the form required for your} model. For photogrammetry, this might include things like camera settings and positions, lighting placement, and preprocessing you did to clean up or organize the source images. For a model created in a solid or surface modeling program, this will include the sources of your data (like museum records, direct measurements, or secondary publications), and any calculations you did on that data. It is especially important to note points where there was uncertainty or conflicting information about your source and how you resolved that.

\section{- The steps you followed and the settings you used in your processing}

programs. Any time you have a choice to make, even if it is keeping a default setting, you want to keep track of it. Once you have a working process, you can probably archive some of the intermediary steps, though you may want to remember why certain promising settings didn't work in case similar questions come up in your model or in review. 


\section{- How much time different parts of the process took and other kinds of data} relating to labor or technology needs. This will help if you need to repeat the project or if you want to apply for grant funding for other project phases. It can also help you decide whether the process is worthwhile for your needs.

- How you exported your model or prepared it for further use. This might include settings, but it could just be what formats you used and whether you needed any intermediate programs to make your project available.

\section{- You also want to keep source content alongside your documentation, or a} note about where you are storing the source data if you have files that are too large to be stored in the same place. This is especially likely to be an issue with photogrammetry, as you may end up with hundreds or thousands of source photos that can take up a lot of room.

Keeping track of all this can get very tedious and interrupt your thinking if you try to do it all in written notes, but there are ways to make documentation less intrusive as you work with liberal use of annotated photos, screenshots, or diagrams. Take photos of your photogrammetry setup and insert them into your main documentation record, with some notes about what they show about distances or conditions. Take screenshots of menu choices and put those in your documentation with notes on the choices you made or the outcomes. Make a list of the source data and add text citations as you would for a paper to keep track of how you got your information. Save versions of your model at each stage so that you can easily go back to an earlier step.

There will likely be other ways that you can streamline documentation for your project, but make it a priority to keep track of what you do. Documentation for dimensional projects is easy to forget because so much of the work happens within programs that are not text based or away from your computer entirely.

\section{Continue Reading: Maintenance \& Sustainability for Dimensional Projects}

